\title{
Procesos de simplificación fonológica en niños de 4, 5 y 6 años con dificultades fonológicas
}

\author{
Phonological simplification processes by children \\ aged 4,5 and 6 years with phonological difficulties
}

\section{María Mercedes Pavez G. \\ Lingüista \\ Profesora Titular, \\ Escuela de Fonoaudiología, Universidad de Chile}

\author{
Carmen Julia Coloma T. \\ Doctora en Psicología, Universidad \\ de Granada \\ Profesora Asociada, \\ Escuela de Fonoaudiologia, \\ Universidad de Chile
}

\section{Mariangela Maggiolo L.}

Fonoaudióloga, Licenciada en Fonoaudiología Profesora Asociada, Escuela de Fonoaudiologia, Universidad de Chile

\section{Christian Peñaloza C.}

Magister en Letras, con Mención en Lingüística, Pontificia Universidad

Católica de Chile

Profesor Asistente,

Escuela de Fonoaudiologia, Universidad de Chie

Contacto con el autor: María Mercedes Pavez Escuela de Fonoaudiología

Facultad de Medicina

Universidad de Chile

Av. Independencia 1027, Santiago

Chile

Tel: (56 2) 29786181

Correo-e: mpavez@med.uchile.cl

\section{RESUMEN}

Se estudia a niños con dificultades fonológicas (DF) desde la perspectiva de la teoría de la fonología natural. Según ella, el desarrollo consiste en la paulatina eliminación de procesos fonológicos de simplificación (PSF) que afectan a la producción de palabras. Los niños con DF no eliminan adecuadamente los PSF y, por ello, sus emisiones son poco inteligibles y similares a la de niños de menor edad. Existe información en español acerca de la eliminación de PSF en el desarrollo típico, pero es escasa en menores con DF. Objetivo: comparar el manejo de PSF en niños con DF de 4, 5 y 6 años. Método: Se trabajó con 34 menores, con DF y diagnóstico de TEL, alumnos de escuela de lenguaje, distribuidos en tres grupos: 4.0 a 4.11 años (n12); 5.0 a 5.11 años (n11) y 6.0 a 6.11 años (n11). Fueron evaluados individualmente en su establecimiento educacional con el Test para evaluar procesos de simplificación fonológica (TEPROSIF-R). Resultados: Se constató que la eliminación de PSF en los niños con problemas fonológicos es evidente entre los 4 y 5 años, pero se lentifica entre los 5 y 6 años, en especial, en los relacionados con la estructura de la sílaba y de la palabra.

Palabras claves: desarrollo fonológico, niños con problemas fonológicos, procesos de simplificación fonológica.

\section{ABSTRACT}

This paper studies children who have phonological difficulties (PD) from the theoretical principles of natural phonology. This theory holds that the development consists in the gradual loss of the phonological simplification processes (PSP) which affect the production of words. Children with PD do not delete properly the PSP and therefore their speech is unintelligible and similar to that of younger children. Studies on the loss of phonological simplification processes in children with typical language development have been carried out in Spanish. However, far too little attention has been paid to this loss in children with PD. Objective: to compare the management of phonological simplification processes by children aged 4,5 and 6 years with PD. Method: 34 children with PD and diagnosed with Specific Language Impairment (SLI), students enrolled in a language school, divided into three groups: 4.0 to 4.11 years (n12); 5.0 to 5.11 years (n11) and 6.0 to 6.11 years (n11). Children were assessed individually in their schools using the Test para Evaluar Procesos de Simplificación Fonológica (TEPROSIF-R). Results: it was observed that the loss of PSP in children with PD is evident between children aged 4 and 5 years, but it is slower between children aged 5 and 6 years, especially, those related to the structure of the syllable and the word.

Key words: phonological development, children with phonological difficulties, phonological simplification processes. 


\section{Introducción}

Los niños con problemas fonológicos se caracterizan, en primera instancia, porque su lenguaje aparece poco inteligible y suele impresionar como el de niños de menor edad. Sin embargo, sus dificultades no son articulatorias ni afectan a la producción de un fonema particular, sino que radican en la emisión de la palabra $\left(\right.$ Bosch $\left.^{1,2}\right)$. Una perspectiva para abordar su alteración es la propuesta por la fonología natural, según la cual los niños simplifican los vocablos mediante estrategias conocidas como procesos de simplificación fonológica (PSF). De acuerdo a este enfoque, el desarrollo fonológico implica la progresiva eliminación de los PSF hasta lograr emitir las palabras según el modelo adulto. Es claro que dicha eliminación coexiste con la adquisición del sistema de fonemas. Los menores con dificultades en el desarrollo fonológico conservan procesos en edades en que ya debieran haberlos eliminado, por eso sus enunciados se asemejan a los de niños más pequeños $^{3,1,2}$. Se caracterizan, por lo tanto, por la persistencia en el tiempo de procesos fonológicos normales en edades tempranas. Es frecuente que problemas de esta índole aparezcan como un síntoma más en el marco de los trastornos específicos del lenguaje.

El concepto de PSF, postulado por la versión original de la teoría como operaciones innatas que actúan sobre la representación fonológica de la palabra, es controvertido ${ }^{4}$. Sin embargo, ha contribuido de modo importante en la comprensión y caracterización tanto de niños con desarrollo típico del lenguaje como de sujetos con dificultades fonológicas ${ }^{1,3,5,6,7,8,9,10,11}$. Incluso se ha utilizado la noción de PSF considerándolos más bien patrones de error ${ }^{12}$ o simplemente reglas de simplificación $^{13}$ útiles para describir el desempeño fonológico de los niños, en particular la discrepancia entre las emisiones infantiles y la producción adulta ${ }^{4}$.

Clásicamente, se distinguen tres grandes tipos de PSF: a) relacionados con la estructura de la sílaba y de la palabra, b) de asimilación y c) de sustitución.

Los procesos relacionados con la estructura de la sílaba y de la palabra son aquellos mediante los cuales el niño tiende a simplificar las sílabas transformándolas en estructuras del tipo consonante + vocal (CV) o las palabras en estructuras CVCV. Los procesos de asimilación son procedimientos donde se cambia un fonema para hacerlo igual o semejante a otro presente en la palabra modelo o en la emitida por el niño. Finalmente, los procesos de sustitución consisten en simplificar el vocablo reemplazando fonemas pertenecientes a una clase por miembros de otra clase. Algunos autores como Grunwell (1985) ${ }^{14}$, adaptado posteriormente por Bosch ${ }^{1,2}$, señalan que los PSF pueden considerarse sistémicos cuando se simplifica el sistema de oposiciones fonológicas como ocurre en las sustituciones. Por otra parte, se consideran estructurales cuando se relacionan con la estructura de la palabra simplificando sílabas, la metría o asimilando fonemas en ella. 
En los niños con desarrollo típico del lenguaje, se ha constatado la progresiva eliminación de PSF entre los 3 y 6 años ${ }^{1,6,7,12,13,15}$. Sin embargo, en menores con trastorno fonológico no está clara la evolución que puede experimentar su manejo de los PSF a medida que aumenta su edad y son escasos los estudios al respecto, en particular en lengua española. Una investigación sobre el tema ha verificado cambios en el perfil fonológico en niños de 3 y 4 años con trastorno específico del lenguaje, hablantes de catalán y español ${ }^{16 .}$ Los procesos más significativos a los 3 años fueron la omisión de sílaba, especialmente inicial, al compararlos con un grupo control pareado por edad. En cambio, los mismos niños a los 4 años presentaban como simplificaciones más características la reducción de secuencias consonánticas y la omisión de consonantes (tanto en el ataque como en la coda silábica), además de ausencia del fonema vibrante múltiple $/ \bar{r} /$. Así, los procesos a los 3 años involucran el nivel de la palabra mientras que a los 4 afectan el nivel silábico y comienza aparecer el nivel segmental que implica fonemas. Lo anterior sugiere una tendencia en el desarrollo que avanzaría desde la palabra hacia la sílaba para finalizar en el sistema fonológico.

De acuerdo a lo ya expuesto, en el presente trabajo el objetivo es comparar el manejo de procesos de simplificación en niños con problemas fonológicos de 4, 5 y 6 años. Todo ello con el propósito de contribuir al conocimiento del desarrollo en hablantes de lengua española con este tipo de dificultad.

\section{Procedimientos metodológicos}

\section{Participantes}

Se trabajó con un grupo de 34 niños distribuidos en tres rangos de edad: 4.0 a 4 años 11 meses (n12); 5.0 a 5 años 5 meses (n11) y 6.0 a 6 años 11 meses (n11).

Todos presentaban dificultades fonológicas y fueron seleccionados en escuelas especiales de lenguaje en Santiago de Chile donde habían sido diagnosticados como niños con Trastornos específico del lenguaje.

El problema fonológico se constató mediante el Test para evaluar procesos de simplificación fonológica, versión revisada, TEPROSIF$\mathrm{R}^{17}$, donde todos los menores presentaron rendimientos deficitarios, esto es, bajo 2 desviaciones estándar con relación al promedio correspondiente a su grupo etario.

EI TEPROSIF- R es una prueba que consta de 37 palabras con distinta metría, acentuación y complejidad silábica, elicitadas mediante imitación diferida y apoyo de láminas (la nómina de palabras se presenta en el Anexo 1). Posee estudios de validez, de confiabilidad y normas para niños chilenos de 3.0 a 6.11 años.

\section{Procedimientos}

Los participantes fueron evaluados individualmente en una sesión, según la normativa del TEPROSIF- R. Para ello, se utilizan las dos primeras láminas como ejemplos para familiarizar al niño con 
la tarea y luego se presentan los ítems propiamente tales. La instrucción consiste en señalar un dibujo en la parte superior de la lámina diciendo al menor una oración que finaliza con la palabra estímulo ("Mira, aquí hay una mariposa"), luego indicando en la parte inferior otro dibujo se enuncia una oración donde falta al final el estímulo evaluado y se pide al niño completarla ("Y ahora, mira acá. En las flores está la...").

Las respuestas se transcribieron fonológicamente y luego se analizaron, según los criterios del test, identificando en ellas 3 grandes tipos de PSF: a) relacionados con la estructura de la sílaba y de la palabra, b) de asimilación y c) de sustitución. Una síntesis con los 3 tipos de PSF y los subprocesos que comprenden se presenta en el Anexo 2.

Antes de realizar el análisis, se constató la confiabilidad inter examinadores con el coeficiente de concordancia W de Kendall. Al tercer intento, se logró un índice de 0.96 (p 0.000) para PSF relacionados con la sílaba, de 0.91 (p 0.000) para los de asimilación y de 0.84 en los de sustitución (p 0.000).

\section{Resultados}

En primer lugar, se calculó el promedio y la respectiva desviación estándar del total de PSF y de cada tipo de ellos en los tres rangos de edad. Luego, para determinar si los grupos diferían estadísticamente entre sí se aplicó un ANOVA. Los resultados obtenidos se aprecian en la Tabla 1, donde se ha agregado el valor del ANOVA junto a los datos descriptivos para ilustrar la comparación (aunque dicho análisis no se realice con los promedios).

Tabla 1. Promedio de PSF utilizados en cada rango de edad por niños con déficit fonológico.

\begin{tabular}{|c|c|c|c|c|c|c|c|}
\hline \multirow[t]{2}{*}{ Rangos de edad } & \multicolumn{2}{|c|}{$\begin{array}{c}4.0-4.11 \text { años } \\
n=12\end{array}$} & \multicolumn{2}{|c|}{$\begin{array}{c}5.0-5.11 \text { años } \\
n=11\end{array}$} & \multicolumn{2}{|c|}{$\begin{array}{c}6.0-6.11 \text { años } \\
n=11\end{array}$} & \multirow[t]{2}{*}{ ANOVA } \\
\hline & PROM & D.E. & PROM & D.E. & PROM & D.E. & \\
\hline Total de PSF & 54.3 & 17.4 & 36.1 & 9.3 & 36.7 & 14.1 & $6.4^{*}$ \\
\hline $\begin{array}{l}\text { Relacionados con } \\
\text { estructura de la silaba } \\
\text { y de la palabra }\end{array}$ & 25.3 & 9.2 & 14.3 & 5.9 & 16.7 & 8.0 & $6.3^{*}$ \\
\hline Asimiliación & 15.7 & 9.5 & 10.8 & 5.1 & 9.7 & 3.7 & 1.6 \\
\hline Sustitución & 12.4 & 3.6 & 11.1 & 4.1 & 9.3 & 4.6 & 2.6 \\
\hline
\end{tabular}

Como se advierte en la Tabla 1, los grupos de niños difieren significativamente en el total de PSF que utilizan y en los procesos relacionados con la estructura de la sílaba y de la palabra. En cambio, no existen diferencias significativas en los PSF de asimilación ni de sustitución.
Para conocer cuáles eran los grupos que diferían, se efectuó un análisis post hoc con la prueba HSD de Tuckey. Se constató que en el total de PSF, son los niños de 4 años los que difieren significativamente de los de 5 (diferencia de medias $18.1^{*}$, p 0.001) con un tamaño del efecto de 1.28 y 
de los de 6 años (diferencia de medias18.5*, p 0.01) con un tamaño del efecto de 1.15. Los niños de 5 y 6 años se comportan de modo similar (diferencia de medias 0.36, p 0.99).

En los procesos relacionados con la estructura de la sílaba y de la palabra, también son los participantes de 4 años quienes difieren de los de 5 (diferencia de medias 11.1*, p 0.006, con un tamaño del efecto de 1.42) y de los de 6 años (diferencia de medias 8.6*, p 0.03, con un tamaño del efecto de 1.0). Entre los 5 y 6 años, en cambio, siguen observándose desempeños semejantes (diferencia de medias 2.4, p 0.74).

En síntesis, en los niños con problemas fonológicos entre los 4 y 5 años hay una significativa disminución en la cantidad de PSF y, en especial, en los relacionados con la estructura de la sílaba y de la palabra, mientras que los desempeños parecen mantenerse sin avances entre los 5 y 6 años.

Posteriormente, se efectuó un análisis más detallado para conocer en qué subprocesos específicos de los relacionados con la estructura de la sílaba y de la palabra se advertían comportamientos distintos entre los grupos etarios estudiados. Se consideraron como procesos que afectan la estructura de la sílaba a la reducción de grupo consonántico, la reducción de diptongo, la omisión de consonante trabante o de coda silábica y la coalescencia. Por otra parte, se identificaron como procesos que afectan a la estructura de la palabra a la omisión de elementos átonos, la omisión de sílaba tónica, la adición de fonemas o sílabas y la inversión de fonemas o sílabas (ver apéndice 2 con ejemplos de cada subproceso).

Se estableció, primero, la frecuencia de uso de los subprocesos en los tres rangos de edad. Dado que el total de PSF de estructura de la sílaba y de la palabra en cada grupo era diferente (4 años 299; 5 años 156 y 6 años 183), se calculó el porcentaje correspondiente a cada subproceso con relación al total. Los resultados se presentan en los Gráficos 1 y 2.

Como se advierte en el Gráfico 1, el proceso relacionado con la estructura de la sílaba más frecuente en los tres grupos fue la omisión de coda silábica o de consonante trabante. En segundo lugar, a los 4 años aparece la reducción de diptongo, a los 5 años la coalescencia y a los 6 años la reducción de grupo consonántico.

Se aplicó luego un ANOVA para conocer la relevancia estadística de las diferencias en estos subprocesos entre los grupos estudiados. Así se observó que existían diferencias significativas solo en la reducción de diptongo ( $\mathrm{F} 4.9 *, \mathrm{p} 0.01)$ y en la omisión de consonante trabante o coda $(\mathrm{F} \mathrm{7.2*,p}$ 0.003). El análisis post hoc con la prueba HSD de Tuckey evidenció que los niños de 4 años en la omisión de trabante difieren significativamente de los de 5 (diferencia de medias 4.05*, p 0.007, con un tamaño del efecto de 1.26) y de los de 6 años (diferencia de medias 3.9*, p 0.008, con un tamaño del efecto 1.29), mientras que no existen diferencias significativas entre 5 y 6 años. En cambio, en la reducción de diptongo se observa una situación curiosa, porque solo los niños de 4 años son los que 
se distinguen significativamente de los de 5 (diferencia de medias $1.3^{*}$, p 0.01, tamaño del efecto
1.37), pero no de los de 6 años. Entre 5 y 6 años, no hay diferencias con significación estadística.

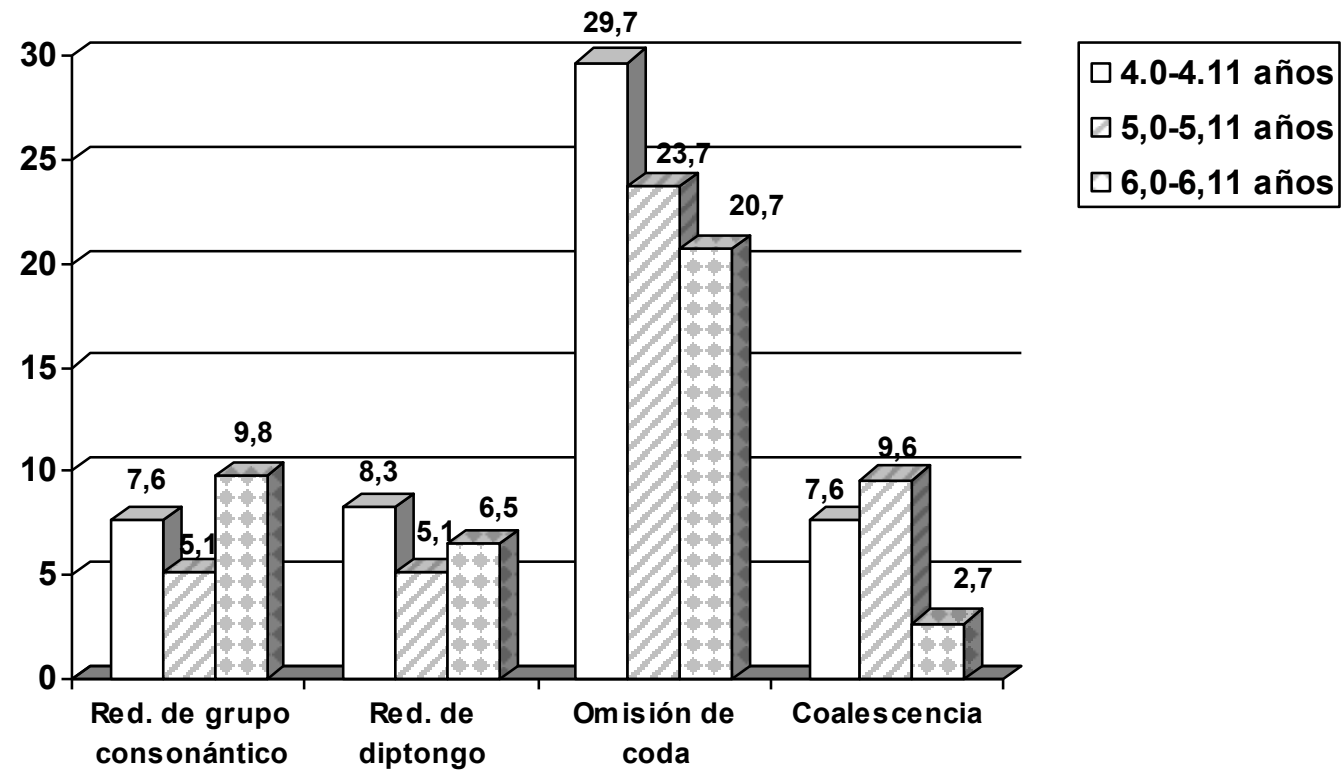

Gráfico 1. Porcentaje de procesos que afectan la estructura de la sílaba en cada grupo etario en niños con dificultades fonológicas.

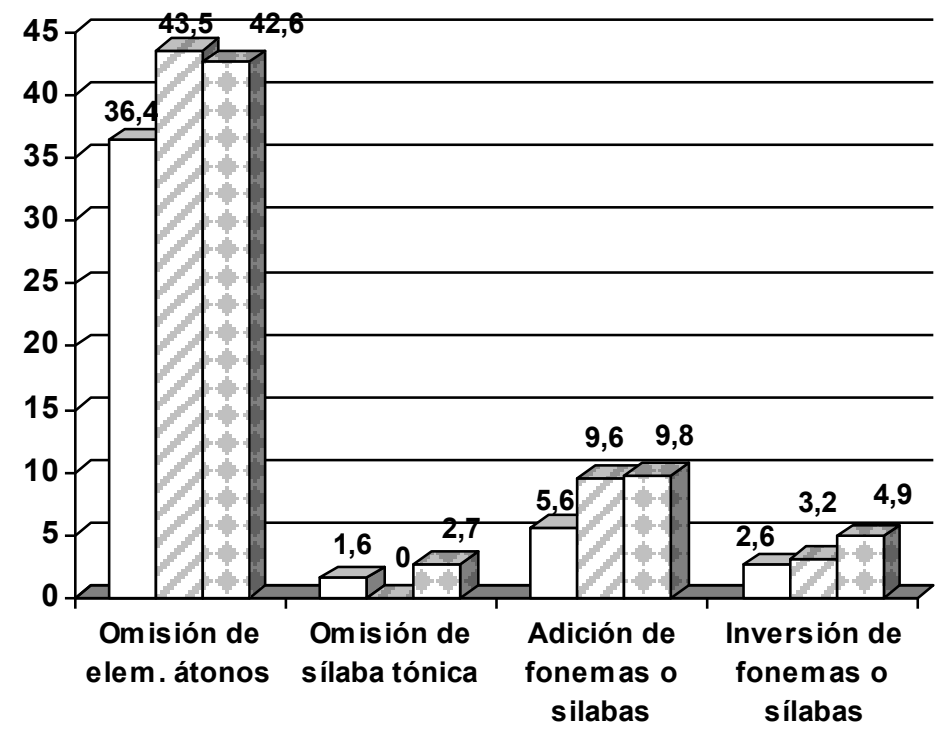

Gráfico 2. Porcentaje de procesos que afectan la estructura de la palabra en cada grupo etario en niños con dificultades fonológicas. 
Entre los procesos que afectan la estructura de la palabra, como se observa en el Gráfico 2, aparece con notoria mayor frecuencia la omisión de elementos átonos en los tres grupos etarios seguido con un porcentaje bastante menor por la adición de fonemas o sílabas.

La comparación mediante un ANOVA permitió observar que no existen diferencias estadísticas entre los tres grupos etarios en ninguno de estos subprocesos.

En síntesis, en los subprocesos que afectan la estructura silábica se advierte que los niños con problemas fonológicos de 4 años manejan significativamente más omisiones de coda que los de 5 y 6 años, mientras que estos últimos poseen desempeños similares. En cambio, a los 4 años solo se usa más reducción de diptongo que a los 5 años. En los subprocesos relacionados con la estructura de la palabra todos los grupos usan mayoritariamente omisión de elementos átonos sin diferir entre sí.

Considerando la variabilidad de rendimientos que puede afectar a los niños con dificultades fonológicas, se decidió complementar la información anterior analizando el desempeño individual en cada rango etario en los subproceso que aparecen con más frecuencia. Para ello, se calculó el porcentaje de niños que usa 3 o más veces cada subproceso. Así, se constató que la omisión de coda es utilizada por el $91,6 \%$ de niños a los 4 años, por $45,4 \%$ a los 5 años y por el 63,6 a los 6 años. La omisión de elementos átonos es empleada por el $83,3 \%$ de niños de 4 años y por el $100 \%$ de los niños de 5 y 6 años.

\section{Discusión}

A partir de los resultados obtenidos, se puede proponer una descripción del desarrollo fonológico en niños de 4, 5 y 6 años con problemas fonológicos. Por cierto, dada la cantidad de participantes ella representa solo una primera aproximación que debiera ser corroborada con investigaciones posteriores.

Al respecto, es posible señalar que a los 4 años existe mayor cantidad de PSF que a los 5 y 6 años, en especial procesos relacionados con la estructura de la sílaba como la omisión de coda y reducción de diptongo, aunque también se observa reducción de grupo consonántico y coalescencia. En simplificaciones que afectan a la palabra, es muy abundante la omisión de elementos átonos.

Por otra parte, a los 5 años el desarrollo fonológico se evidencia en un significativo menor uso de PSF en relación a los 4 años (aunque todavía a niveles deficitarios), se adquiere un mayor manejo de la estructura silábica con coda y con diptongo. Se mantiene sin cambios significativos la reducción de grupos consonánticos y la coalescencia. No se advierte progreso en el manejo de la estructura de la palabra donde continúa estable la omisión de elementos átonos.

Por último, a los 6 años no se observan cambios en el desarrollo fonológico con relación a los 5 años. En consecuencia, sus características fonológicas son muy semejantes a la de los niños de 5 años. Incluso, persiste la reducción de diptongo observada a los 4 años. 
Se constata así que la eliminación de procesos de simplificación en los niños con problemas fonológicos es evidente entre los 4 y 5 años, pero parece lentificarse entre los 5 y 6 años donde no se advierten diferencias significativas. Ello podría indicar que el desarrollo fonológico es menor entre estas últimas edades y que las dificultades tienden a ser más persistentes. Es importante destacar que el mayor desarrollo se relaciona en especial con simplificaciones que afectan la estructura de la sílaba y de la palabra que disminuyen a los 5 años. Los procesos de sustitución, que se vinculan con el manejo del sistema fonológico, no difieren en los tres grupos etarios estudiados. En cuanto a los procesos de asimilación, que suelen ser considerados estructurales $^{13,1,2,14}$ tampoco se observan diferencias. Si se enfatiza que las asimilaciones implican armonizar los fonemas en la palabra, podría señalarse que los desempeños más relacionados con unidades fonémicas se mantienen sin cambios relevantes.

Así, los niños con dificultades fonológicas probablemente mejoran a los 5 años su manejo más bien a nivel estructural que sistémico comparados con los de 4.

Lo anterior es concordante con la tendencia advertida en niños de 3 y 4 años con trastorno específico del lenguaje según la cual primero se observan simplificaciones que afectan a la palabra, luego se evoluciona a procesos que reducen la sílaba. Por último, se utilizan simplificaciones a nivel de fonemas $^{16}$.
El hecho de que la omisión de la coda silábica sea significativamente más frecuente en los niños de 4 años con dificultades fonológicas también corrobora los hallazgos previos en niños de esa edad al ser comparados con los de 3 años. Aparece así como un fenómeno característico que disminuye a los 5 y 6 años. Sin embargo, no ocurre lo mismo con la reducción de grupos consonánticos en que los tres rangos etarios estudiados no difieren. Es decir, la omisión de la coda evoluciona disminuyendo de los 4 a 5 y 6 años (donde su uso se mantiene). En cambio, la reducción de grupo consonántico que implica simplificar el ataque silábico complejo persiste sin modificaciones relevantes en los niños con dificultades fonológicas en esas edades. Así, el manejo de la coda parece lograrse antes que el del ataque complejo con secuencias consonánticas.

Respecto a esto último, se ha señalado que las dos primeras etapas que caracterizan la adquisición de los grupos consonánticos homosilábicos son la elisión del fonema líquido y luego la sustitución de este fonema por yod (/pjáto/ por $/$ pláto $/)^{18}$. Se advierte, por lo tanto, que los menores con problemas fonológicos estudiados conservan hasta edades avanzadas estrategias para simplificar las sílabas correspondientes a etapas iniciales de la adquisición de la estructura silábica.

Algo similar se observa en las simplificaciones que afectan a la estructura de la palabra donde los grupos etarios no difieren entre sí y el proceso más frecuente es la omisión de elementos átonos (generalmente pretónicos). Esta estrategia es común también en el desarrollo fonológico inicial y corrobora la persistencia de 
simplificaciones tempranas. En relación con ello, se ha constatado en niños con trastorno específico del lenguaje de 4 años el uso de estrategias similares a las utilizadas por menores de 2 años. Dichas estrategias consisten en omitir sílabas átonas iniciales que dejan una huella acústica de la sílaba suprimida (lo que se evidencia en una mayor longitud en la palabra reducida). Este desempeño correspondería a un primer paso para luego incluir la totalidad de las sílabas en la palabra ${ }^{19}$. En el presente estudio, la omisión de la sílaba átona se mantiene incluso hasta los 6 años.

Por ende, los niños con problemas fonológicos experimentan cambios en su desempeño, pero mantienen dificultades básicas con la estructuración de la palabra incluso a los 6 años conjuntamente con limitaciones en la organización silábica. Su desarrollo, por lo tanto, se caracteriza por la persistencia de estrategias de simplificación que afectan la estructuración fonológica. Sería recomendable explorar más sobre el desempeño fonológico de niños con este tipo de problemas en español y así contribuir con información que puede ser útil para su abordaje terapéutico y educativo.

Fuente de financiamiento: Proyecto SOC $06 / 17-2$ de la Vicerrectoría de Investigación y Desarrollo, Universidad de Chile.

\section{Referencias}

1. Bosch, L. (2003). Trastornos del desarrollo fonético y fonológico. En M. Puyuelo y J. A. Rondal (editores) Manual de desarrollo y alteraciones del lenguaje. Barcelona, Masson.189 - 204.

2. Bosch, L. (2004). Evaluación fonológica del habla infantil. Barcelona, Masson.

3. Ingram, D. (1983). Trastornos fonológicos en el niño. Barcelona, Médica y Técnica.

4. Galeote, M. (2002) Adquisición del lenguaje. Madrid, Pirámide.

5. Acosta, V.; Moreno, A.; Ramos, V.; Quintana, A. y Espino, O. (1996). La evaluación del lenguaje, Granada, Aljibe.

6. Goldstein, B. e Iglesias, A. (1996). Phonological Patterns in Normally Developing Spanish - Speaking 3- and 4Years- Olds of Puerto Rican Descent. Language, Speech, and Hearing Services in Schools, Vol.27, 82 - 90.

7. Goldstein,B. e Iglesias, A. (1996). Phonological Patterns in Puerto Rican Spanish-Speaking Children with Phonological Disorders. J. Commun. Disor. 29, 367-387.

8. Acosta, V., León, S-. y Ramos, V. (1998). Dificultades del habla infantil: un enfoque clínico. Málaga: Aljibe.

9. Acosta, V. y Moreno, A. (1999). Dificultades del lenguaje en ambientes educativos. Barcelona, Masson.

10. Storti, P. (2002). Estudio descriptivo sobre los Procesos fonológicos de simplificación en niños de 2 años a 5 años. Tesis para obtener el grado de licenciado en Fonoaudiología, Universidad Nacional de Rosario.

11. Villegas, F. (2004). Manual de logopedia, evaluación e intervención de las dificultades fonológicas. Madrid, Pirámide.

12. Dodd, B.; Holm, A.; Hua, Z. y Crosbie, S. (2003). Phonological development: a normative study of British English children. Clinical Linguistics \& Phonetics, 17, no 8, 617- 643.

13. Serra, M., Serrat, E., Solé, R., Bel, A. y Aparici, M. (2000). La adquisición del lenguaje. Barcelona, Ariel Psicología. 
14. Grunwell, P. (1985). Phonological Assessment of Child Speech (PACS). Windsor: NFER- Nelson.

15. Pavez, M.M.; Maggiolo, M. ; Peñaloza, C. y Coloma, C. J. (2009). Desarrollo fonológico en niños de 3 a 6 años: incidencia de la edad, el género y el nivel socioeconómico. Revista de Lingüística Teórica y Aplicada (RLA), 47(2), 89 109.

16. Aguilar-Mediavilla, E. y Serra-Raventós, M. (2006). Phonological Profile of Spanish-Catalan Children with Specific language Impairment at Age 4: Are There Any Changes over Time? Folia Phoniatr Logop. 58, 400 414.
17. Pavez, M.M.; Maggiolo, M. y Coloma, C.J. (2009). Test para evaluar procesos de simplificación fonológica, versión revisada, TEPROSIF-R., Ediciones Universidad Católica de Chile, Santiago, $3^{\circ}$ edición actualizada.

18. Oropeza, M. (2001). Adquisición de la estructura silábica del español en niños de dos a seis años. Colección Pedagógica Universitaria, 36, 1-13.

19. Carter, A. y Gerken, L. (2003). Similarities in weak syllable omissions between children with specific language impairment and normally developing language: a preliminary report. Journal of Communication Disorders, 36, 165 - 179. 
Anexo 1

Las 37 palabras utilizadas en el TEPROSIF-R son:

$\begin{array}{ll}\text { 1. plancha } & \text { 14. remedio } \\ \text { 2. rueda } & \text { 15. peineta } \\ \text { 3. mariposa } & \text { 16. auto } \\ \text { 4. bibicleta } & \text { 17. indio } \\ \text { 5. helicóptero } & \text { 18. pantalón } \\ \text { 6. bufanda } & \text { 19. camión } \\ \text { 7. Caperucita } & \text { 20. cuaderno } \\ \text { 8. alfombra } & \text { 21. micro } \\ \text { 9. refrigerador } & \text { 22. tren } \\ \text { 10. edificio } & \text { 23. plátano } \\ \text { 11. calcetín } & \text { 24. jugo } \\ \text { 12. dinosaurio } & 25 . \text { enchufe } \\ \text { 13. teléfono } & 26 . \text { jabón }\end{array}$

27. tambor

28. volantín

29. jirafa

30. gorro

31. árbol

32. dulce

33. guitarra

34. guante

35. reloj

36. jaula

37. puente

12. dinosaurio

26. jabón 


\section{Anexo 2}

Síntesis de procesos de simplificación fonológica TEPROSIF-R.

\section{PROCESOS RELACIONADOS CON LA ESTRUCTURA DE LA SÍLABA Y DE LA PALABRA (E).}

E.1. Reducción de grupo consonántico

E.2. Reducción de diptongo

E.3. Omisión de consonante trabante o de coda silábica

E.4. Coalescencia

E.5. Omisión de elementos átonos

E.6.Omisión de sílaba tónica o de alguno de sus constituyentes

E.7. Adición de fonemas o sílabas

E.8. Inversión de fonemas o sílabas

\section{PROCESOS DE ASIMILACIÓN (A).}

\section{A.1.ASIMILACIÓN IDÉNTICA}

ASIMILACIONES POR SEMEJANZA :

A.2. Labial

A.3 Dental

A.4 Palatal

A.5 Velar

A.6. Asimilación a fonemas líquidos

A.7. Asimilación nasal

A.8. Asimilación vocálica

A.9 Asimilación silábica
: / tren/ /t_en/

:/puénte/ /p_énte/

: /pantalón/ /pa_talón/

:/ remédio/ / reméŷo/

:/alfómbra/ /_fómbra/

/maripósa/ /ma_pósa/

:/maripósa/ /marí_sa /

/elikóptero/ /eli_óp tero/

:/ refrixeradór/ / refrixexeradór/

/plátano/ /plántano/

:/teléfono/ /tenéfolo/ :/plátano/ /plátamo/

:/maripósa/ /madipósa/

:/kuadérno/ /kuaŷérno/

: /bufánda/ /gufánda/

: /gitára/ /litára/

:/alfómbra/ /anfómbra /

: /alfómbra / /elfómbra/

: /elikóptero/ /lilikóptero/

\section{PROCESOS DE SUSTITUCIÓN (S).}

PROCESOS QUE AFECTAN A FONEMAS QUE TRABAN LA SÍLABA (CODA SILÁBICA).

S.1.Aspiración de fonema trabante de sílaba

:/dúlse/ /dúhse/. 
PROCESOS SEGÚN ZONA DE ARTICULACIÓN.

S.2. Posteriorización (de labiales y dentales)

S.3. Frontalización (de palatales y velares)

$$
\begin{aligned}
& \text { :/bufánda/ /ĉufánda/ } \\
& \text { :/guánte/ / buánte/. }
\end{aligned}
$$

S.4. Labialización de consonantes (que no son palatales, velares ni líquidos) :/dinosáurio/ /binosáurio/.

PROCESOS SEGÚN MODO DE ARTICULACIÓN.

S.5. Oclusivización de fonemas fricativos o africados (de zona similar conservando el rasgo áfono o sonoro): /xiráfa / /kiráfa/.

S.6. Fricativización de fonemas oclusivos o africados (de zona similar conservando el carácter áfono o sonoro): /puénte/ /fuénte/.

S.7. Fricativos entre sí : /alfómbra/ /alsómbra/.

PROCESOS SEGÚN FONACIÓN O CUALIDAD ARTICULATORIA.

S.8. Sonorización de consonantes (conservando la zona) : : / /kaperusíta/ /kaberusíta/.

S.9. Afonización o pérdida de sonoridad de consonantes (conservando la zona) : / refrixeradór/ / refrixeratór/. PROCESOS SEGÚN RASGO FUNDAMENTAL: FONEMAS LÍQUIDOS / NO LÍQUIDOS.

S.10. Semiconsonantización de fonemas líquidos

S.11 Sustitución de fonemas líquidos entre sí

$$
\begin{aligned}
& \text { :/plátano/ /pjátano/. } \\
& \text { :/tambór/ /tamból/. }
\end{aligned}
$$

S.12.Sustitución de fonemas líquidos por no líquidos orales: /góro/ /gódo/; /xáula/ /xáuba/.

S.13. Sustitución de fonemas no líquidos orales o vocálicos por líquidos: /edifísio/ /elifísio/. PROCESOS SEGÚN RESONANCIA SUPLEMENTARIA.

S.14. Nasalización de fonemas

S.15. Oralización de consonantes nasales
: /ruéda/ /muéda/

: /bufánda/ /bufálda/

PROCESOS QUE AFECTAN A LOS FONEMAS VOCÁLICOS.

S.16. Sustitución de vocales o disimilación

:/puénte/ /puénta/ 